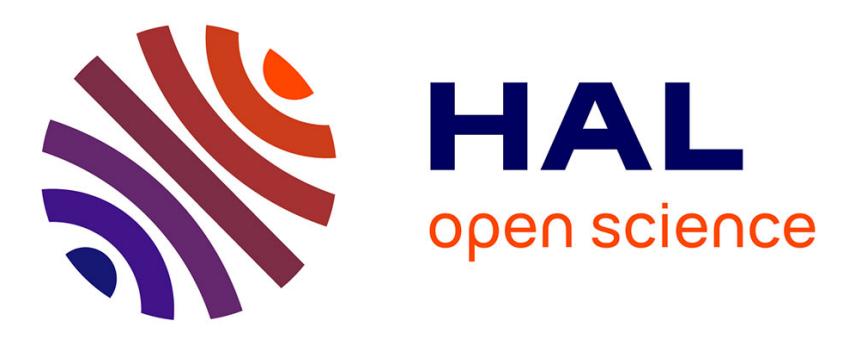

\title{
Analyse du fonctionnement des chambres à étincelles à échantillonnage
}

\author{
G. Metzger, F. Schmitt, J. Gresser, M. Riedinger, G. Sutter
}

\section{To cite this version:}

G. Metzger, F. Schmitt, J. Gresser, M. Riedinger, G. Sutter. Analyse du fonctionnement des chambres à étincelles à échantillonnage. Revue de Physique Appliquée, 1970, 5 (5), pp.771-776. 10.1051/rphysap:0197000505077100 . jpa-00243453

\section{HAL Id: jpa-00243453 https://hal.science/jpa-00243453}

Submitted on 1 Jan 1970

HAL is a multi-disciplinary open access archive for the deposit and dissemination of scientific research documents, whether they are published or not. The documents may come from teaching and research institutions in France or abroad, or from public or private research centers.
L'archive ouverte pluridisciplinaire HAL, est destinée au dépôt et à la diffusion de documents scientifiques de niveau recherche, publiés ou non, émanant des établissements d'enseignement et de recherche français ou étrangers, des laboratoires publics ou privés. 


\title{
ANALYSE DU FONCTIONNEMENT DES CHAMBRES A ÉTINCELLES A ÉCHANTILLONNAGE
}

\author{
par G. METZGER, F. SCHMITT, J. GRESSER, M. RIEDINGER, G. SUTTER \\ Centre de Recherches Nucléaires de Strasbourg
}

(Reçu le 25 juillet, révisé le 4 mars et 25 mai 1970)

\begin{abstract}
Résumé. - Le fonctionnement des chambres à étincelles à échantillonnage est analysé par le calcul de l'efficacité en fonction de la tension et du délai et par le calcul du temps de formation de l'étincelle. bon.

Dans la limite des hypothèses de base, l'accord entre les valeurs expérimentales et calculées est
\end{abstract}

Abstract. - By use of the theory of the spark formation of Raether the efficiency versus high voltage and delay and the spark formation time of sampling spark chambers have been calculated. The comparison with the experimental results is good within the limits of the basic hypothesis.

1. Introduction. - Les chambres à étincelles à échantillonnage sont intensivement utilisées depuis une dizaine d'années dans les expériences de physique des particules.

Cependant très peu d'auteurs [1] [2] [3] [4] [5] se sont intéressés à l'analyse du fonctionnement des chambres en vue de la déduction de relations utilisables pour la prévision des caractéristiques. Il faut remarquer néanmoins Maurel [4] qui donne une théorie de l'efficacité en fonction du délai dont les résultats sont bien vérifiés par l'expérience ainsi que le calcul du temps de formation effectué par Fischer et Zorn [5]. L'étude de l'efficacité en fonction de la tension reste par contre au stade où l'avait menée Bella et Franzinetti [1].

Notre propos est de reprendre ces divers calculs en les basant sur le mécanisme de formation de l'étincelle dit "streamer mecanism " de Loeb, Meek et Raether [6], [7], [8] d'où nous tirons les trois éléments de base de notre calcul de l'efficacité :

- Il existe un stade critique, qui, étant atteint, nous donne avec certitude une étincelle.

- Ce stade critique peut se caractériser par un nombre donné de porteurs.

- Dans le cas des chambres à étincelles il y a distribution de l'ionisation primaire le long de la trajectoire. Chaque électron primaire démarrant une avalanche, il est nécessaire de tenir compte de l'interaction exercée par ces avalanches. Cette dernière remarque est fondamentale en ce sens qu'elle détermine la difficulté d'établissement d'un calcul dans le cas des chambres à étincelles.
2. Calcul de l'efficacité en fonction de la tension $(\boldsymbol{\eta}=\boldsymbol{f}(\boldsymbol{V}))$. - a. PRINCIPE. - Nous considérons l'efficacité unitaire des chambres à étincelles donnée par la

$$
\eta=\frac{\text { nombre d'étincelles }}{\text { nombre de déclenchements }} \text {. }
$$

Un critère d'alignement est introduit dans le dépouillement des données expérimentales pour rejeter toute étincelle erratique.

En conséquence l'efficacité est la probabilité pour qu'une avalanche au moins, atteigne le stade critique. La formule de Furry-Wisjman [9] [10] permet alors le calcul de l'efficacité en fonction de $\alpha$ (premier coefficient de Townsend). En effet soit $V(n, x)$ la probabilité pour qu'une avalanche démarrée par $n_{0}$ électrons atteigne un nombre de porteurs $n$

$$
V(n, x)=\frac{1}{n_{0}} \exp -\alpha x\left(1-\frac{1}{n_{0}} \exp -\alpha x\right)^{n-1}
$$

alors l'efficacité est :

$$
\begin{aligned}
\eta & =\sum_{n=N}^{\infty} V(n, x) \\
& =\left[1-\frac{1}{n_{0}} \exp -\alpha x\right]^{N-1}
\end{aligned}
$$

$N=$ nombre d'électrons dans une avalanche au stade critique.

Comme $N=10^{8}, N-1 \simeq 10^{8}$ et l'efficacité devient

$$
\eta \simeq\left[1-\frac{1}{n_{0}} \exp -\alpha d\right]^{N}
$$

$d=$ distance interélectrode. 
Il reste à introduire l'expression donnant $\alpha$ en fonction de la tension $V$, ou du champ électrique $E(V / d)$, pour obtenir l'efficacité en fonction de la tension. Aucune relation théorique générale pour $\alpha=f(V)$ et $\alpha=f(E)$ n'existe à l'heure actuelle.

Les courbes de la figure 1 montrent que dans un assez grand domaine des équations du type

$$
\begin{aligned}
\alpha & =A p \exp -\frac{B p}{E} \\
\log \alpha & =a+\frac{b V}{p d}
\end{aligned}
$$

expliquent assez bien les résultats expérimentaux.

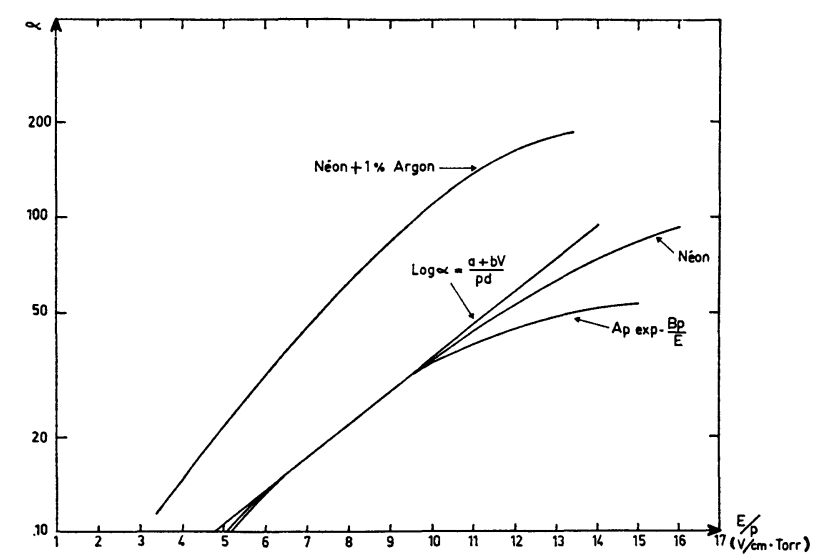

Fig. 1. - Approximations de $\alpha=f(E / p)$.

b. Calcul. - Les relations précédentes sont basées sur l'existence de $n_{0}$ électrons primaires au niveau de la cathode (Fig. $2 a$ ), alors que réellement il existe $v$ électrons distribués dans l'entreplaque.

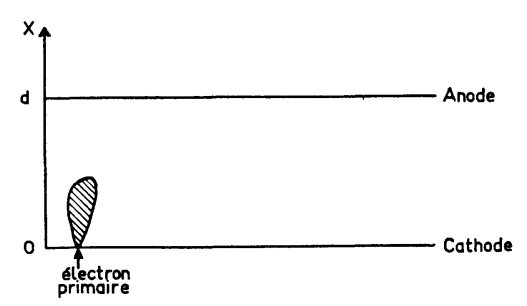

FIG. $2 a$. - Définition des équivalences.

En première approximation de l'interaction des avalanches, nous proposons comme Maurel de remplacer la définition précédente de l'efficacité par: l'efficacité est la probabilité pour que le nombre total d'électrons dans l'entreplaque atteigne le nombre critique $N$.

Ceci posé, la figure $2 b$ montre la répartition des électrons primaires, $\bar{v}$ étant l'ionisation spécifique, et nous constatons que le nombre d'électrons pouvant être créé par les électrons situés près de l'anode est bien inférieur à celui que les électrons primaires situés près de la cathode peuvent engendrer. Les paramètres $d_{u}$

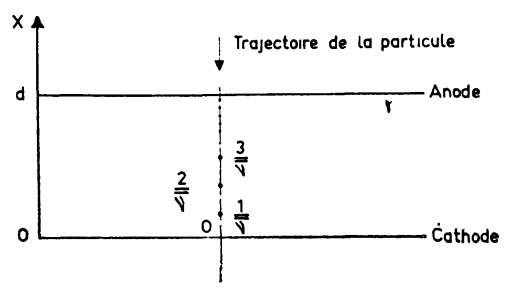

FIG. $2 b$.

et $n_{u}$ traduisent cette remarque. Ils sont définis par les relations :

$$
\exp \alpha d_{u}=100
$$

(100 fois moins d'électrons créés). Soit :

$$
d_{u}=\frac{4,6}{\alpha} \quad \text { et } \quad n_{u}=\bar{v} d_{u} .
$$

D'autre part, en supposant pour les $n_{u}$ électrons primaires distribués sur le segment $d_{u}$ une interaction nulle des avalanches, nous pouvons définir une abscisse équivalente $x_{e}$ par les relations :

$$
\begin{aligned}
& \bar{N}_{\text {total }}=\exp \alpha\left(d-\frac{1}{\bar{v}}\right)+\exp \alpha\left(d-\frac{2}{\bar{v}}\right)++\cdots+\exp \alpha\left(d-\frac{n_{u}}{\bar{v}}\right) \\
&+\cdots \\
&= \exp \alpha\left(d-\frac{1}{\bar{v}}\right)\left[\frac{1-\exp -\frac{\alpha n_{u}}{\bar{v}}}{1-\exp -\frac{\alpha}{\bar{v}}}\right] \\
&=n_{u} \exp \alpha\left(d-x_{e}\right) .
\end{aligned}
$$

Ceci revient à considérer que $n_{u}$ électrons primaires localisés en $x_{e}$ font sur la distance $d-x_{e}$ le même effet que $n_{u}$ électrons primaires distribués sur $d_{u}$ (Fig. 2c). En explicitant le calcul, on peut donner la relation approchée :

$$
x_{e} \simeq \frac{1}{\bar{v}}+\frac{1,5}{\alpha} .
$$

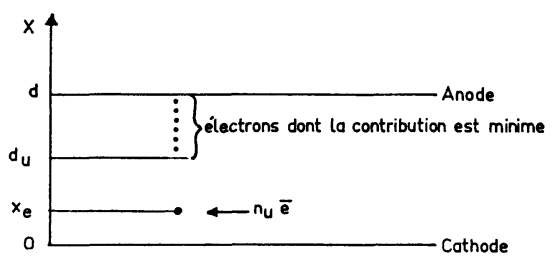

Fig. $2 c$.

Compte tenu des résultats du paragraphe a), l'efficacité en fonction de $\alpha$ est alors donnée par :

$$
\eta=\left[1-\frac{\exp -\alpha\left(d-x_{e}\right)}{n_{u}}\right]^{10^{8}} .
$$


L'efficacité en fonction de la tension s'obtient simplement en explicitant $\alpha=f(E)$ comme en a).

c. ConsÉQUenCES. - L'expression précédente de l'efficacité traduit celle-ci en fonction de $\alpha$, donc en fonction de la tension, mais également en fonction de la distance interélectrode $d$, de la pression p par l'intermédiaire de $\alpha=f(p)$ et de $\bar{v}=f(p)$ et de l'ionisation spécifique $\bar{v}$.

d. Discussion Des hyPothèses. DOMAINE DE VAliDITÉ. - Les hypothèses principales du calcul précédent sont :

1) La théorie de Raether est applicable aux chambres à étincelles.

2) Le nombre critique de porteurs est $N=10^{3}$.

3) La croissance des avalanches est " relativement " indépendante de la présence d'autres avalanches.

D'autres hypothèses, telles que la validité des équations reliant $\alpha$ au champ électrique, la forme de l'impulsion électrique, etc... sont secondaires.

En effet, l'hypothèse [1] semble être exacte ainsi que le prouve le fonctionnement des chambres à « avalanches » et à "streamers ». D'autre part, un calcul d'erreur simple montre que si $N$ varie entre $10^{7}$ et $10^{9}$ l'erreur relative sur la détermination des points d'efficacité $10 \%$ et $90 \%$ n'est que de $5 \%$.

Par contre, l'hypothèse [3] est fondamentale, et notre modification de la définition de l'efficacité en donne qu'une première approximation.

En conséquence le calcul doit fournir des résultats corrects dans les cas où l'ionisation spécifique $\bar{v}$ est faible et où $\alpha$ atteint la valeur critique pour des champs élevés, et des résultats de plus en plus faux dans les cas où $\bar{v}$ est grand et où $\alpha$ atteint la valeur critique pour des champs faibles entraînant une forte interaction des avalanches.

Il est évident alors que les autres hypothèses sont secondaires, dans la mesure cependant où la durée de l'impulsion de haute tension est suffisante pour permettre le développement de l'étincelle, et que son temps de montée ainsi que son délai sont suffisamment courts pour ne pas observer un déplacement important de la distribution des électrons primaires.

e. ApProximations PRATIQUes. - L'utilisation pratique de la relation de l'efficacité en fonction de la tension sous la forme générale :

$\eta=\left\{1-\frac{1}{n_{u}}\left[\exp -\left(\operatorname{Ap} \exp -\frac{B p d}{V}\right)\left(d-x_{e}\right)\right]\right\}^{10^{8}}$

est cependant longue. Aussi l'approchons-nous par les relations (Fig. 3) :

$$
\begin{aligned}
& \alpha_{0}=\frac{18,52-\log n_{u}}{d-x_{e}} ; \\
& \quad \alpha_{1}=\frac{20,72-\log n_{u}}{d-x_{e}} ; S_{\alpha}=0,364\left(d-x_{e}\right)
\end{aligned}
$$

$$
\begin{aligned}
& V_{0}=\frac{-B p d}{\log \alpha_{0}-\log A p} ; \\
& \quad V_{1}=\frac{-B p d}{\log \alpha_{1}-\log A p} ; S_{v}=S_{\alpha} \cdot \alpha \frac{b}{p d}
\end{aligned}
$$

donnant les points intéressants : $\alpha$ et tension correspondant à une efficacité de $10 \%\left(\alpha_{0}, V_{0}\right)$ de $90 \%$ $\left(\alpha_{1}, V_{1}\right)$; pente $S_{\alpha}$ et $S_{v}$ de la transition supposée linéaire des courbes $\eta=f(\alpha), \eta=f(V)$.

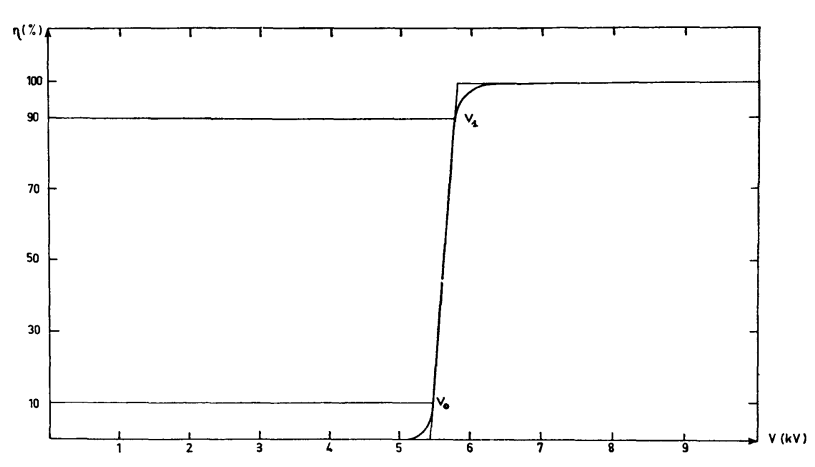

Fig. 3. - Efficacité calculée. Approximations.

f. RÉSultats eXPÉRIMENTAuX. Comparaison a la THÉORIE. - Deux ensembles de chambres à échantillonnage ont été construits. Le premier comporte dix chambres d'entreplaque $1 \mathrm{~cm}$, les plaques épaisses ne permettant le passage qu'à des particules cosmiques. Le deuxième peut être traversé par des électrons (source de ${ }^{90} \mathrm{Sr}$ ), les électrodes étant des feuilles minces d'acier inoxydable de $25 \mu \mathrm{m}$ ou d'Aluminium de $15 \mu \mathrm{m}$; l'entreplaque étant de $5 \mathrm{~mm}$ et l'empilement comportant 3 à 5 chambres.

La figure 4 montre les résultats expérimentaux de l'efficacité en fonction de la tension, comparés aux courbes calculées. Nous constatons que dans le cas de l'Hélium ( $\bar{v}$ faible et $\alpha$ critique atteint pour un champ moyen), l'accord entre le calcul et l'expérience est correct. Par contre, cet accord est moins bon dans le cas du Néon. En effet, $\bar{v}_{\mathrm{Ne}}=6 \bar{v}_{\mathrm{He}}$ et $\alpha$ critique est atteint pour un champ faible rendant l'hypothèse [3] invalide.

Dans le cas de l'Argon, l'accord calcul-expérience est retrouvé malgré $\bar{v}_{A}=13 \bar{v}_{\mathrm{He}}$, puisque pour ce gaz $\alpha$ critique est atteint pour un champ élevé et par conséquent l'hypothèse [3] reste valide.

Enfin la figure 5 présente nos courbes calculées en variant $\bar{v}_{A}$ d'un facteur 75 correspondant selon Grieder [11] à l'ionisation spécifique du rayonnement $\alpha$ émis par le ${ }^{210}$ Po. Les courbes expérimentales de Grieder sont également reproduites. La figure 5 met ainsi en évidence la limite de validité de notre calcul en montrant que l'hypothèse [3] est bien essentielle et que notre approximation est encore insuffisante. Ce cas correspond en effet à $\bar{v}$ très grand $\left(\bar{v}_{A}\right.$ pour le $\left.{ }^{210} \mathrm{P}_{\mathrm{o}} \simeq 1000 \bar{v}_{\mathrm{He}}\right)$ et la grande valeur du champ pour $\alpha$ critique ne suffit plus à compenser cette grande 

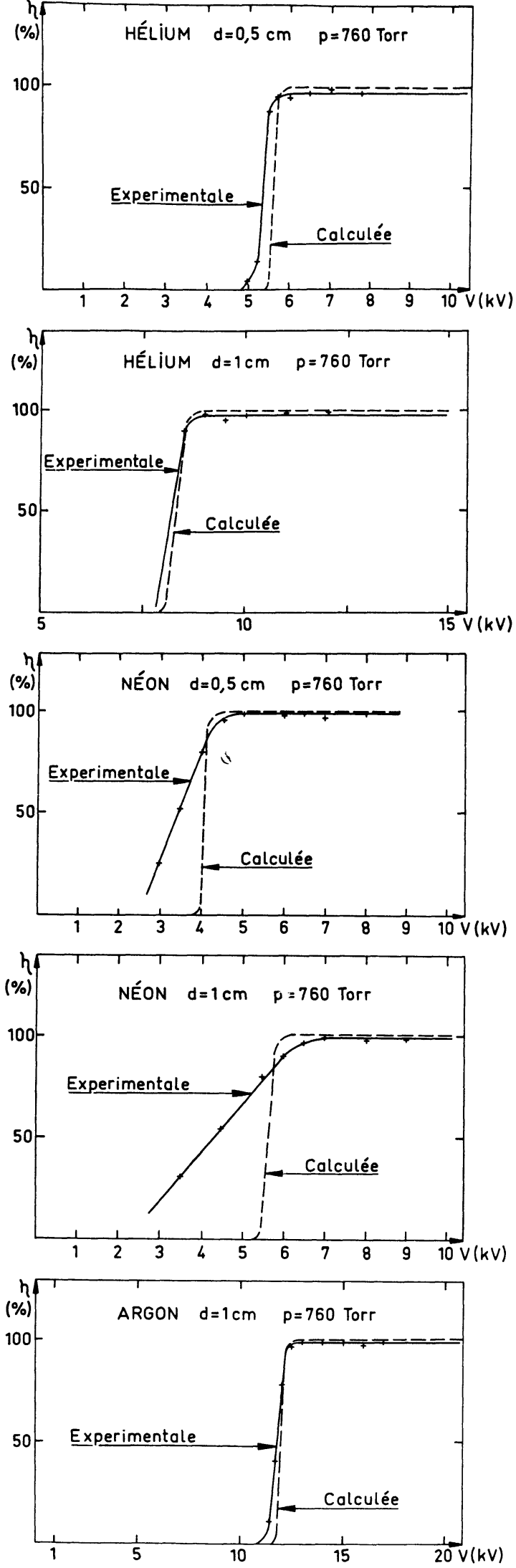

FIg. 4. - Efficacité en fonction de la tension : Résultats expérimentaux et courbes calculées.

ionisation spécifique pour garder l'hypothèse [3] valable. Cependant, il est clair que le calcul donne

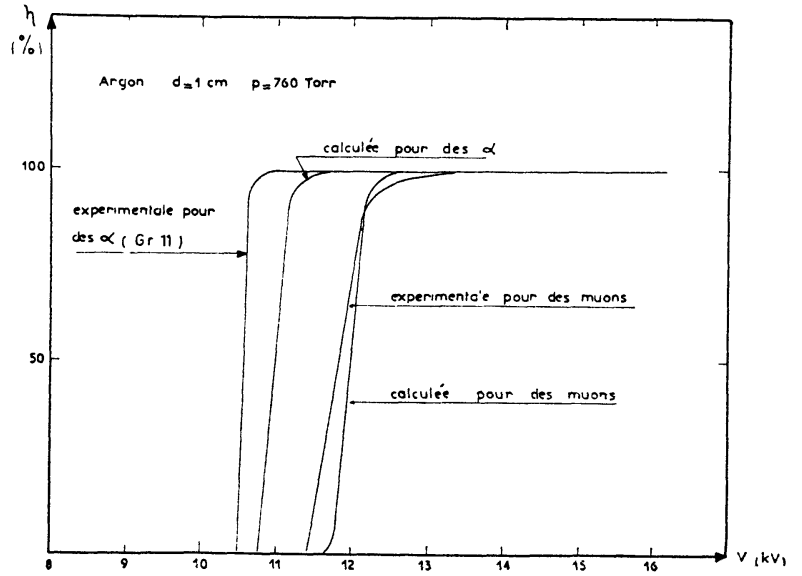

Fig. 5. - Efficacité en fonction de la tension pour 2 valeurs d'ionisation spécifique.

un déplacement minimum des courbes, et permet donc une prévision « pessimiste».

On peut ainsi remarquer sur la figure 5 la possibilité d'une nouvelle propriété des chambres à échantillonnage: la sélection des particules selon le pouvoir ionisant, l'intérêt de notre calcul étant de permettre une prévision limitée de l'incidence des divers paramètres.

3. Efficacité en fonction du délai. - Le paramètre pratique intéressant des courbes d'efficacité en fonction du délai, est la longueur $t_{d p}$ du palier. Cette longueur se détermine aisément en considérant que l'efficacité reste unité tant qu'une avalanche au moins peut dégénérer en streamer.

Ceci implique la notion de « distance critique 》 $d_{\mathrm{c}}$ à laquelle doit se trouver un électron primaire au moins.

Il vient : $d_{\mathrm{c}}$ défini par $\exp \alpha \cdot V_{D}(E) \cdot t=10^{8}$

où

$$
\alpha . V_{D}\left(E_{b}\right) t=d_{c} \text {. }
$$

Soit

$$
t_{d p}=-\frac{\log 10^{8}}{\alpha \cdot V_{D}\left(E_{b}\right)}
$$

$V_{D}\left(E_{b}\right)$ est la vitesse de dérive des électrons dans le gaz sous l'effet du champ de balayage $E_{b}$.

Ou encore

$$
t_{d p} \simeq \frac{19}{\alpha \cdot V_{D}\left(E_{b}\right)}
$$

Cette relation est très analogue à l'expression obtenue par Maurel [4] et est bien vérifiée par l'expérience ainsi que le montre le tableau $\mathrm{I}$.

Remarquons que dans le cas des courbes d'efficacité en fonction du délai, les hypothèses du paragraphe précédent sont valables, en particulier celle de l'indépendance de la croissance des avalanches. En effet le champ de balayage déplace les électrons primaires, et, combiné au délai, fait diminuer très for- 
tement leur nombre jusqu'à l'amener à une valeur voisine de l'unité.

4. Calcul du temps de formation $(\pi)$ de l'étincelle. - A partir de la notion de distance critique introduite précédemment, le temps de formation est :

$$
\tau=\frac{d_{\mathrm{c}}}{\alpha \cdot V_{D}}+\tau_{1}
$$
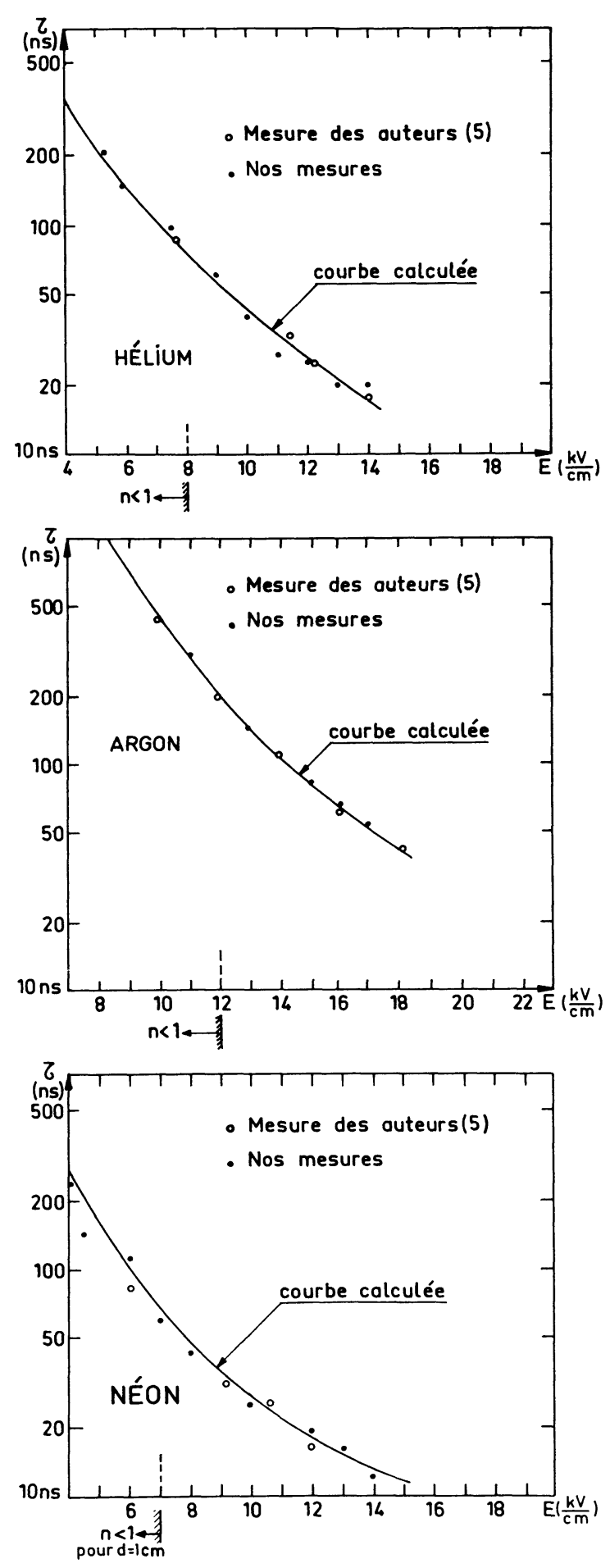

Fig. 6. - Temps de formation de l'étincelle, expérimental et calculé. où $V_{D}$ est la vitesse de dérive des électrons sous l'effet du champ électrique de l'impulsion et $\tau_{1}$ le temps de formation du canal de décharge, le stade du streamer étant atteint.

En introduisant les équivalences définies au paragraphe $2 b$ il vient

$$
\tau \simeq \frac{\log 10^{8}-\log n_{u}}{\alpha \cdot V_{D}}+\tau_{1} .
$$

La valeur $\tau_{1}$ peut être calculée, approximativement par la relation

$$
\tau_{1} \simeq \frac{d}{10^{8}}
$$

en admettant que la vitesse moyenne d'établissement du canal de décharge soit de $10^{8} \mathrm{~cm} / \mathrm{s}$ [8].

Il vient alors :

$$
\tau \simeq \frac{\log 10^{8}-\log n_{u}}{\alpha \cdot V_{D}}+\frac{d}{10^{8}} .
$$

La figure 6 donne les courbes calculées par cette relation ainsi que nos points expérimentaux. Certains relevés de Fischer et Zorn [5] sont également reportés. Il faut signaler que ces auteurs sont les premiers à avoir effectué un calcul du temps de formation de l'étincelle. Leur relation, basée sur l'existence d'un électron unique ne tient pas compte du temps d'établissement du canal de décharge, le stade du streamer étant atteint. Il en résulte dans certains cas (Néon) un désaccord entre la théorie et l'expérience, que notre calcul permet d'éviter.

5. Conclusions. - La théorie de Raether de la formation d'une étincelle conduit donc à des relations permettant de prévoir dans une grande mesure les caractéristiques d'une chambre à étincelles à échantillonnage.

L'introduction de certains paramètres: nombre utile $n_{u}$, distance utile $d_{u}$, abscisse équivalente $x_{e}$, permet de simplifier les expressions théoriques pour obtenir des relations utilisables en pratique. Ceci dans le domaine de validité des hypothèses simplificatrices, en particulier celle de l'indépendance de la croissance des avalanches. Les résultats expérimentaux confirment la plupart des déductions théoriques, en particulier, on peut remarquer que :

- la pureté du gaz de remplissage des chambres est un facteur prédominant pour l'obtention de résultats reproductibles;

- le calcul des courbes d'efficacité en fonction de la tension $\eta=f(V)$ est valable dans les cas courants (He, $A$ à la pression atmosphérique). Cependant lorsque l'ionisation spécifique augmente ( $\mathrm{Ne}$ ou cas de particules fortement ionisantes) et lorsque $\alpha$ varie fortement avec la tension la pente calculée de la transition de l'efficacité devient fausse, ceci étant dû à une forte interaction des avalanches. Le seuil 
d'efficacité $90 \%\left(V_{1}\right)$ donne des résultats quantitatifs dans les cas usuels et des résultats qualitatifs assez corrects dans les autres cas;

- le calcul de la longueur du palier des courbes d'efficacité en fonction du délai $n=f\left(t_{D}\right)$ est en bon accord avec les résultats expérimentaux, car l'hypothèse de l'indépendance de la croissance des avalanches devient de plus en plus valable en fonction du délai ;

- l'introduction des paramètres $n_{u}$ et $\tau_{1}$ permet une prévision correcte du temps de formation de l'étincelle.

De plus, il apparaît la possibilité de discrimination des particules traversant une chambre à étincelles en fonction de leur pouvoir ionisant.

Enfin la théorie de l'efficacité d'une chambre a pu être étendu au calcul de l'efficacité d'un éclateur.

\section{TABLEAU I}

Longueurs $t_{d p} d u$ palier d'efficacité $100 \%$, calculées et mesurées

a) NÉON.

$$
d=1 \mathrm{~cm}
$$

$E_{b}(V / \mathrm{cm}) \ldots \ldots \ldots \ldots, 0$

$\boldsymbol{t}_{d p}$ Calculé $(\mu \mathrm{s}) \quad \ldots \ldots \ldots$

$t_{d p}$ Mesuré $(\mu \mathrm{s}) \ldots \ldots \ldots$

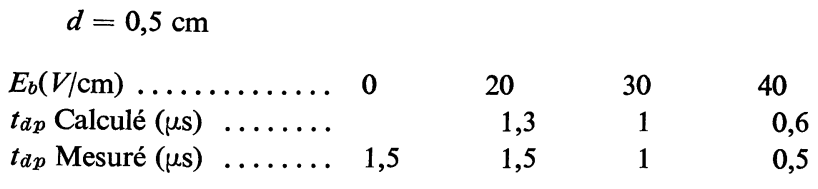

\section{b) HÉLIUM}

$$
d=1 \mathrm{~cm}
$$

$\begin{array}{lllrcc}E_{b}(V / \mathrm{cm}) \ldots \ldots \ldots \ldots & 0 & 20 & 40 & 80 \\ t_{d p} \text { Calculé }(\mu \mathrm{s}) & \ldots \ldots \ldots & & 8 & 1,2 & 0,8 \\ t_{d p} \text { Mesuré }(\mu \mathrm{s}) & \ldots \ldots \ldots & 9 & 8 & 1,4 & 1\end{array}$

$$
d=0,5 \mathrm{~cm}
$$

$\begin{array}{lllll}E_{b}(V / \mathrm{cm}) \ldots \ldots \ldots \ldots & 0 & 30 & 50 \\ t_{d p} \text { Calculé }(\mu \mathrm{s}) & \ldots \ldots \ldots & & 2,8 & 1,9 \\ t_{d p} \text { Mesuré }(\mu \mathrm{s}) & \ldots \ldots \ldots & 7 & 2,6 & 1,6\end{array}$

c) Argon

$$
d=1 \mathrm{~cm}
$$

$E_{b}(V / \mathrm{cm}) \ldots \ldots \ldots \ldots, 0$

$t_{d p}$ Calculé $(\mu \mathrm{s}) \ldots \ldots \ldots$

$t_{d p}$ Mesuré $(\mu \mathrm{s}) \ldots \ldots \ldots 2$

\section{Bibliographie}

[1] Bella (F.) et Franzinetti (C.), Nuovo Cimento, 1953, 10, 1335, 1338, 1461.

[2] Cranshaw (T. E.) et De Beer (J. F.), Nuovo Cimento, $1957,5,110$

[3] Bayukov (Y. D.), N. I. M., 1963, 20, 198.

[4] Maurel (J.), Ségur (P.), Trapp (J. P.) et Blanc (D.), N. I. M., 1966, 42, 173 .

[5] Fischer (J.) et ZORN (G. T.), R. S. I., 1961, 32, 499.

[6] LoEB (L. B.), “ Fundamental Processes of Electrical Discharge in Gases )) New York, 1939.
[7] Meek (J. M.) et Graggs (J. D.), ( Electrical Breakdown of Gases ") Oxford Un. Press (Clarendon) London, 1953.

[8] Raether (H.), “ Electron Avalanches and Breakdown in Gases ) London Butterworth, 1964.

[9] Furry (W. H.), Phys. Rev., 1937, 52, 569.

[10] Wisjman (R. A.), Phys. Rev., 1949, 75, 833.

[11] Grieder (P. K. F.), R. S. I., 1966, 37, 80. 